\title{
PHYSICS OF HIGH-MOLECULAR MATERIALS
}

$\mathrm{T}$ HE concept of a high polymer as a substance composed of molecules of chain-like form built up by the continuous addition of one or more simple units is now so familiar that it is difficult to realize how recent are the scientific developments by which its validity has been established. In the case of rubber, for example, although Faraday had established its empirical formula as early as 1826, and Joule and Kelvin had discussed its unusual thermoelastic properties in the middle of the century, the question whether its molecular weight was of the order of 1,000 or of the order of $1,000,000$ was still under discussion less than twenty years ago. However, since that time, and particularly since the revolutionary change in viewpoint brought about by the emergence of the statistical approach to the problems of polymer chemistry and polymer physics, associated particularly with the names of $K$. $H$. Meyer, W. Kuhn, P. J. Flory, H. Mark and E. Guth, progress in this field has been extraordinarily rapid, and the impact of this progress on industrial technology has been a factor of major economic importance.

The occasion of the Silver Jubilee Symposium on the "Physical Properties of Polymers" held in London during April 15-17 to mark the twenty-fifth anniversary of the formation of the Plastics and Polymer Group of the Society of Chemical Industry provides a suitable opportunity to look back and survey the changes which have taken place during this period and also to attempt to assess the trend of development at the present time.

Polymer science may be said to be a product of colloid chemistry, and it is natural that in the earlier stages of its development the problems of the determination of the chemical structure of polymers dominated the field. However, it soon became apparent that the interpretation of their physical properties presented a challenge of equal scientific interest, so that to-day this side of the subject is attracting an increasing amount of attention. Indeed, the outstanding impression created by the current phase of development is the wide range of variation of properties which may be achieved by changing the physical state of a polymer of a given basic chemical composition, by modifying the conditions of polymerization so as to control the degree of chain regularity, branching, etc., and hence the susceptibility to crystallization.

Basically, polymers may be divided into three broad groups. These are first, the soft amorphous polymers or rubbers; secondly, the hard amorphous polymers or organic glasses; and thirdly, the (partially) crystalline polymers, which have physical properties intermediate between the rubbers and the glasses. Each of these classes represents a particular physical state, and these states are closely analogous to the corresponding liquid, glassy (supercooled liquid) and crystalline states of low-molecular materials. While it is known that many polymers (including natural rubber) may by suitable treatment be brought into two and sometimes all three of these different states, it has nevertheless, until recently, been practically useful to regard any given chemical type as belonging effectively to one or other of these classes. With the introduction of new types of catalyst (Ziegler cata. lysts), however, this classification is beginning to break down, for not only is the range of crystallinity capable of considerable modification (as in polyethylene and polypropylene) but also substances such as polystyrene which have hitherto been thought of as essentially amorphous may be brought into the crystalline state. These 'isotactic' polymers are believed to owe their properties to a perfectly regular sequence of 'head-to-tail' addition of monomer units in a single chain, as distinct from the random arrangement, together with chain branching, which follows from the more usual processes. Another factor which may affect the physical properties of a polymer, either through its effect on crystallinity or otherwise, is the degree of cross-linking. To effect cross-linking by chemical means it is necessary that the polymer chain should contain a suitable reactive site for chemical attack. Materials which are fully saturated or non-reactive cannot be cross-linked chemically without the introduction of a second component, which is frequently a disadvantage. It has been found, however, that in many cases cross-linking may be effected by subjecting the pure polymer to radiation in an atomic pile, or to high-speed electron bombardment. Polyethylene treated in this way shows a continuous reduction of crystallinity with increasing cross-linking, and a corresponding gradation of physical properties.

It appears from these and other studies that an investigation of the molecular factors which determine the tendency to crystallize, and of methods of estimating the degree of crystallinity in polymers, is likely to assume considerable practical importance. In a paper on this topic presented to the recent Sym. posium by C. W. Bunn (Imperial Chemical Industries, Ltd.) this subject is critically reviewed, and the possibilities of newer techniques, such as the infra-red method and nuclear magnetic resonance, for estimating crystallinity, are considered. Bunn also refers to the important question of the 'texture' of the polymer, involving such considerations as the size, orientation and mutual arrangement of the crystallites, which may have an important bearing on physical properties, but about which very little is known at present.

From the structural point of view the soft amorphous polymers or rubbers are the simplest and most fully understood class of materials, at least in respect of their reversible or equilibrium elastic behaviour, which is accounted for in a reasonably satisfactory quantitative manner by the statistical theory. The degree of refinement which has been achieved in this field is well illustrated by a contribution from D. W. Saunders (British Rayon Research Association), dealing with the application of the laws of photo-elasticity derived from the statistical theory to a number of rubber-like polymers. In recent years, however, attention has shifted from the purely elastic behaviour of rubbers to their visco-elastic or time-dependent phenomena, which assume importance in conditions of dynamic loading (vibration phenomena, etc.).

In this province there is at present no general molecular theory available, and it is necessary to fall 
back on more empirical methods of approach. The most usual type of treatment, illustrated by a number of contributions to the Symposium, is related to the method of 'reduced variables' introduced by Ferry and his associates. According to this method the response of the material to an oscillating stress (for example, dynamic modulus and loss angle) is studied over as wide a range of frequency as possible, and at a number of temperatures. It is then found that a change of frequency is equivalent to a change of temperature (the precise relation between these two variables depending on the material), and hence it is possible to construct a single 'master curve' which may be regarded as representing the properties of the material over the whole of the range of variables covered. This set of data should then, in principle, enable the behaviour of the material under any arbitrary conditions of stress and temperature to be predicted. In the Symposium the application of this method, not only to the mechanical properties but also to the formally analogous electrical properties, was discussed by A. R. Payne (Research Association of British Rubber Manufacturers). Other contributors emphasized the limitations of the method, particularly when large strains are encountered, and in crystalline materials, where more complex structural changes appear to accompany the application of even quite small stresses.

Although not based on a molecular theory, the study of dynamic properties by these methods may nevertheless reveal, through the overall pattern of the so-called 'relaxation spectrum', regions of transition which can be associated, at least tentatively, with specific molecular processes. Notable among these are the region corresponding to the transition from the rubber-like to the glassy state, which is present in both crystalline and amorphous polymers, and the region in which melting of the crystallites occurs. Other transitions indicating the onset of a restricted rotation or local movement of side groups may also appear, and the following up of such phenomena by systematic variation of the chemistry of the polymer, as in the work of E. A. W. Hoff and W. Reddish (Imperial Chemical Industries), opens up interesting possibilities.

From the more immediately practical contributions to the Symposium an impression is gained of the increasingly severe range of requirements which plastics and other high polymers are expected to satisfy. Of particular interest are the 'expanded' light-weight materials required in the aircraft industry, and for thermal insulation at very low temperatures; these were discussed by G. C. Ives and J. A. Mead (Dr. V. E. Yarsley (Plastics Testing Laboratories), Ltd.). Another important practical property, dealt with by G. Hulse (Monsanto, Ltd.), is resistance to shock or impact; this is a subject in which the specification of a suitable method of testing may present formidable difficulties. In the electrical industry, the need for dielectrics capable of withstanding high voltages calls for much work in the study of the initiation of discharges and methods of estimating durability under a variety of operating conditions; this field of inquiry was treated in contributions by N. Parkman (Electrical Research Association) and by G. C. Ives and M. M. Riley (Dr. V. E. Yarsley (Plastics) Testing Laboratories, Ltd.).

From the various lines of work referred to in this brief review it is clear that the range of polymer materials available both for scientific study and for practical utilization continues to expand at an increasing rate, and that there is no sign at present that this process is in any sense approaching saturation. If it should continue in the next twenty-five years as it has in the last, the possibilities are, to say the least, interesting.

L. R. G. TreLoaB

\title{
UTILIZING THE NATURAL RESOURCES OF LAKE VICTORIA FOR THE BENEFIT OF FISHERIES AND AGRICULTURE
}

\author{
By R. S. A. BEAUCHAMP \\ East African Fisheries Research Organization, Jinja, Uganda
}

\begin{abstract}
A SHORTAGE of sulphates in solution is a factor affecting the productivity of Lake Victoria. This was discovered by growing cultures of algae in known amounts of lake water to which were added solutions containing all but one of the essential plant nutrients and then observing the growth made in these various media ${ }^{1}$. The usual method for determining chemical deficiencies by observing the reduction of chemical substances in solution and relating them to the growth of the plankton is not applicable in Lake Victoria, because under the tropical conditions obtaining, seasonal fluctuations in the plankton are slight and because throughout the year the amount of sulphate in the Lake water is too low to be readily detectable by chemical means.

As sulphates are present in most waters of the world in quite high concentrations, the discovery that they were a limiting factor in Lake Victoria was a matter of considerable interest.

A study of the records made by water analysts in different areas of Africa revealed that in many African lakes and streams sulphates are present in
\end{abstract}

only very small amounts ${ }^{2}$; I suggested that this might indicate that sulphates were in short supply in the soils through which these streams drain. Several agricultural departments in East Africa have since found that many soils in Uganda, Kenya and Tanganyika contain only a little sulphate; apart from references to this in current annual reports, their results have not yet been published. It seems, moreover, that in some cases where fertilizer trials with ammonium sulphate and superphosphates have led to increased production of crops, part of the increased yield has been due to the sulphate contained in these fertilizers. Applications of gypsum in the Kitale area of Kenya have led to increased yields, particularly of leguminous plants used for fodder. Although all these investigations are only in an early stage, there are clear indications that many African soils contain too little sulphate for maximum production.

So far as the work of the East African Fisheries Research Organization was concerned, it was obviously of great importance to investigate the sulphur cycle 